\title{
Lavado peritoneal laparoscópico como tratamiento de diverticulitis aguda perforada*
}

\author{
Drs. NICOLÁS PEREIRA C. ${ }^{1}$, JAVIER VEGA S..$^{2}$ ALEJANDRO READI V. ${ }^{1}$, MARIO ABEDRAPO M. ${ }^{1}$
}

1 Departamento de Cirugía, Hospital Clínico Universidad de Chile.

2 Mutual de Seguridad. Cámara Chilena de la Construcción.

Santiago, Chile.

\section{Abstract}

\section{Laparoscopic peritoneal lavage as treatment for acute perforated diverticulitis}

Introduction: Sigmoid diverticular disease is common in the occidental world and it is responsible for a great number of hospitalizations. The prevalence of diverticular disease increases with age and only in few cases it is manifest as diverticulitis. Diverticulitis produces variable clinical manifestations, ranging from simple inflammation that only requires medical management, to a life threatening condition, due to a free perforation that requires urgent surgery. Clinical case: In this article we present a clinical case of complicated diverticulitis with free perforation treated with laparoscopic peritoneal lavage.

Key words: Acute diverticulitis, laparoscopic peritoneal lavage.

\section{Resumen}

Introducción: La enfermedad diverticular es muy común en el mundo occidental y es responsable de un gran número de hospitalizaciones. La prevalencia de la enfermedad diverticular aumenta con la edad y sólo en pocos casos se presenta como diverticulitis. La diverticulitis produce manifestaciones clínicas variables que van desde la simple inflamación que sólo requiere tratamiento médico, a una condición potencialmente mortal, debido a una perforación libre que requiere cirugía de urgencias. Caso clínico: Se presenta un caso clínico de diverticulitis complicada con perforación libre manejado con lavado peritoneal laparoscópico.

Palabras clave: Diverticulitis aguda, lavado peritoneal laparoscópico.

\section{Introducción}

La diverticulitis de sigmoides es una enfermedad muy frecuente en el mundo occidental y es responsable de un gran número de hospitalizaciones ${ }^{1}$. La prevalencia de la enfermedad diverticular aumenta con la edad, llegando a un $66 \%$ en mayores de 85 años ${ }^{2}$, y sólo entre el 10 y $25 \%$ de los pacientes con diverticulosis presentan un episodio de diverticulitis ${ }^{3}$. El espectro de la diverticulitis va desde un episodio único de inflamación leve de manejo ambulatorio hasta una peritonitis generalizada de riesgo vital causada por la perforación diverticular aguda que requiere cirugía de urgencias.

*Recibido el 24 de junio de 2012 y aceptado para publicación el 11 de septiembre de 2012.

Los autores no refieren conflictos de interés.

Correspondencia: Dr. Nicolás Pereira C.

Santos Dumont 999, Santiago, Chile.

nicolaspereirac@gmail.com 
La diverticulitis se presenta generalmente con dolor abdominal, típicamente localizado en el cuadrante inferior izquierdo y asociado, en menor o mayor medida, a signos de irritación peritoneal. La fiebre y el alza en el recuento leucocitario orientan al diagnóstico.

Es apropiado clasificar la diverticulitis en diferentes categorías, ya que la morbilidad y mortalidad de esta condición es muy variable. Tradicionalmente, la clasificación de Hinchey ha sido usada para subdividir la diverticulitis aguda complicada en subgrupos basado en el grado y extensión del proceso inflamatorio-infeccioso.

Se llama enfermedad diverticular complicada a aquella que resulta como consecuencia de la inflamación diverticular. Dentro de ésta, se incluyen procesos crónicos como fístula y estenosis, o condiciones agudas, ya sea flegmón, abscesos y perforación libre con peritonitis purulenta y/o fecaloídea. En general, se recomienda el tratamiento quirúrgico para la diverticulitis aguda complicada después del primer episodio debido a que un tercio presentara un segundo episodio ${ }^{4}$. Sin embargo, el manejo no quirúrgico puede ser apropiado en un grupo seleccionado de pacientes si la edad o comorbilidades médicas no permiten una cirugía segura ya que la necesidad de cirugía de urgencias es poco probable 5 .

A continuación se presenta un caso de diverticulitis aguda perforada tratado mediante lavado peritoneal laparoscópico.

\section{Caso clínico}

Paciente sexo masculino de 78 años, con antecedentes de hipertensión arterial en tratamiento e insuficiencia renal crónica en hemodiálisis, prostatectomía radical con linfadenectomía por cáncer de próstata y enfermedad diverticular difusa.

En septiembre de 2011 consulta en el Servicio de Urgencia por un cuadro de 3 días de evolución caracterizado por dolor abdominal en flanco y fosa ilíaca izquierda, náuseas, vómitos y fiebre. Ingresa bien hidratado, febril, hemodinámicamente estable y taquicárdico, destacando al examen físico un abdomen en tabla y signos de irritación peritoneal. En los exámenes de laboratorio destaca una hemoglobina de $9,1 \mathrm{gr} / \mathrm{dL}$, hematocrito de $27,6 \%$, leucocitos de $2.120 / \mathrm{mm}^{3}$ con $20 \%$ de baciliformes, PCR de $393 \mathrm{mg} / \mathrm{L}$ y ácido láctico de $2,7 \mathrm{mmol} / \mathrm{L}$. Se toman hemocultivos y se realiza una Tomografía Computada (TC) de abdomen y pelvis sin contraste donde se evidencia líquido libre de moderada cuantía. Se realiza una paracentesis diagnóstica dando salida a líquido turbio que se envía a estudio.
Se decide el ingreso a pabellón de Urgencias con el diagnóstico de peritonitis difusa de probable origen diverticular, para la realización de una laparoscopía exploradora. Durante la intervención quirúrgica se visualiza gran cantidad de líquido libre seropurulento con zonas de fibrina a nivel del colon sigmoides. Asociado a esto, a nivel de la pelvis se identifica un absceso que se lava y aspira. A la exploración dirigida del colon sigmoides se pesquisa un divertículo indurado, friable, que al comprimirlo da salida a líquido purulento. Se toman cultivos, se realiza un aseo con abundante solución fisiológico y una colorrafia con Vicryl® 3-0. Se coloca un drenaje tubular en la fosa ilíaca izquierda.

El paciente es trasladado a la unidad de cuidados intensivos (UCI) bajo cobertura antibiótica de amplio espectro (Tazonam + Amikacina + Vancomicina). Evoluciona con disfunción orgánica múltiple, sin complicaciones desde el punto de vista quirúrgico. Se ajusta el esquema antibiótico a Ceftriaxona + Metronidazol debido a la presencia de E.Coli multisensible en los hemocultivos y cultivos de líquido peritoneal, para completar 14 días de tratamiento. Evoluciona favorablemente con parámetros inflamatorios a la baja y disfunción multiorgánica en resolución, por lo que al día 14 de postoperatorio egresa de la UCI. Se retira el drenaje al día 18 y se da de alta con control ambulatorio luego de un mes de hospitalización, siendo programado para sigmoidectomía electiva.

\section{Discusión}

El caso clínico presentado, representa el primer reporte publicado a nivel nacional de este tipo de manejo de la diverticulitis aguda perforada. El manejo a través de lavado laparoscópico asociado a tratamiento antibiótico endovenoso aparentemente tiene una baja tasa de morbilidad, baja mortalidad, corta estadía hospitalaria y puede ser realizada sin necesidad de colostomía. Otras ventajas comparadas con la operación de Hartmann son el menor tiempo quirúrgico y menores costos económicos. Así, el lavado laparoscópico sin resección de sigmoides en el cuadro agudo de peritonitis purulenta por una diverticulitis perforada puede ser considerado como una alternativa terapéutica válida a procedimientos más radicales que incluyen la operación de Hartmann. Sin embargo, es necesario de manera minuciosa especificar las indicaciones pre e intraoperatorias, y si la resección electiva de colon se debe realizar a todos o algunos pacientes seleccionados en el seguimiento, para lo cual se necesitan ensayos clínicos randomizados antes que se puedan establecer indicaciones precisas al respecto. 


\section{Referencias}

1. Etzioni DA, Mack TM, Beart RW Jr, Kaiser AM. Diverticulitis in the United States: 1998-2005: changing patterns of disease and treatment. Ann Surg. 2009;249:2107.

2. Parks TG. Natural history of diverticular disease of the colon. Clin Gastroenterol. 1975;4:53-69.
3. Waugh JM, Walt AJ. Current trends in the surgical treatment of diverticulitis of the sigmoid colon. Surg Clin North Am. 1962;42:1267-76.

4. Stollman N, Raskin JB. Diverticular disease of the colon. Lancet 2004;363:631-9.

5. Nelson RS, Ewing BM, Wengert TJ, Thorson AG. Clinical outcomes of complicated diverticulitis managed nonoperatively. Am J Surg. 2008;196:969-72. 